$9=0$

\title{
Where do they come from, where are they going? The importance of socioeconomic rehabilitation of individuals with Alcohol Use Disorder.
}

\author{
Teixeira, J1;:Moutinho, L2; Ramos, R. ${ }^{3} ;$ Ferreira, S. ${ }^{4}$
}

\begin{abstract}
1 MD; Psychiatrist at Unidade de Tratamento e Reabilitação Alcoologica (UTRA); Centro Hospitalar Psiquiátrico de Lisboa (CHPL); joanateixeira@chpl.min-saude.pt 2 Nurse Specialist in Mental Health and Psychiatry at UTRA, CHPL; Master in Cognitive Behavioral Therapies; Doctor of Nursing; lidiamoutinho@chpl.min-saude.pt 3 Social Worker at UTRA, CHPL; ritaramos@chpl.min-saude.pt

4 Clinical Psychologist at UTRA, CHPL; Master in Forensic Psychology and Social Exclusion; Family and Systemic Therapist; soniaferreira@chpl.min-saude.pt
\end{abstract}

Background: The consequences of alcohol consumption to the individual as well as to the society in which he is inserted are well known. Treatment success is associated with the socioeconomic rehabilitation of individuals as a means of consolidating the abstinence achieved during hospitalization. To achieve this it is important to know the socioeconomic situation of individuals who seek treatment for Alcohol Use Disorder.

Objectives: To characterize the socio-demography of a sample of hospitalized individuals during the year 2018 in an inpatient unit of alcohology during the hospital stay.

Materials and Methods: Application of a socio-demographic questionnaire to the patients.
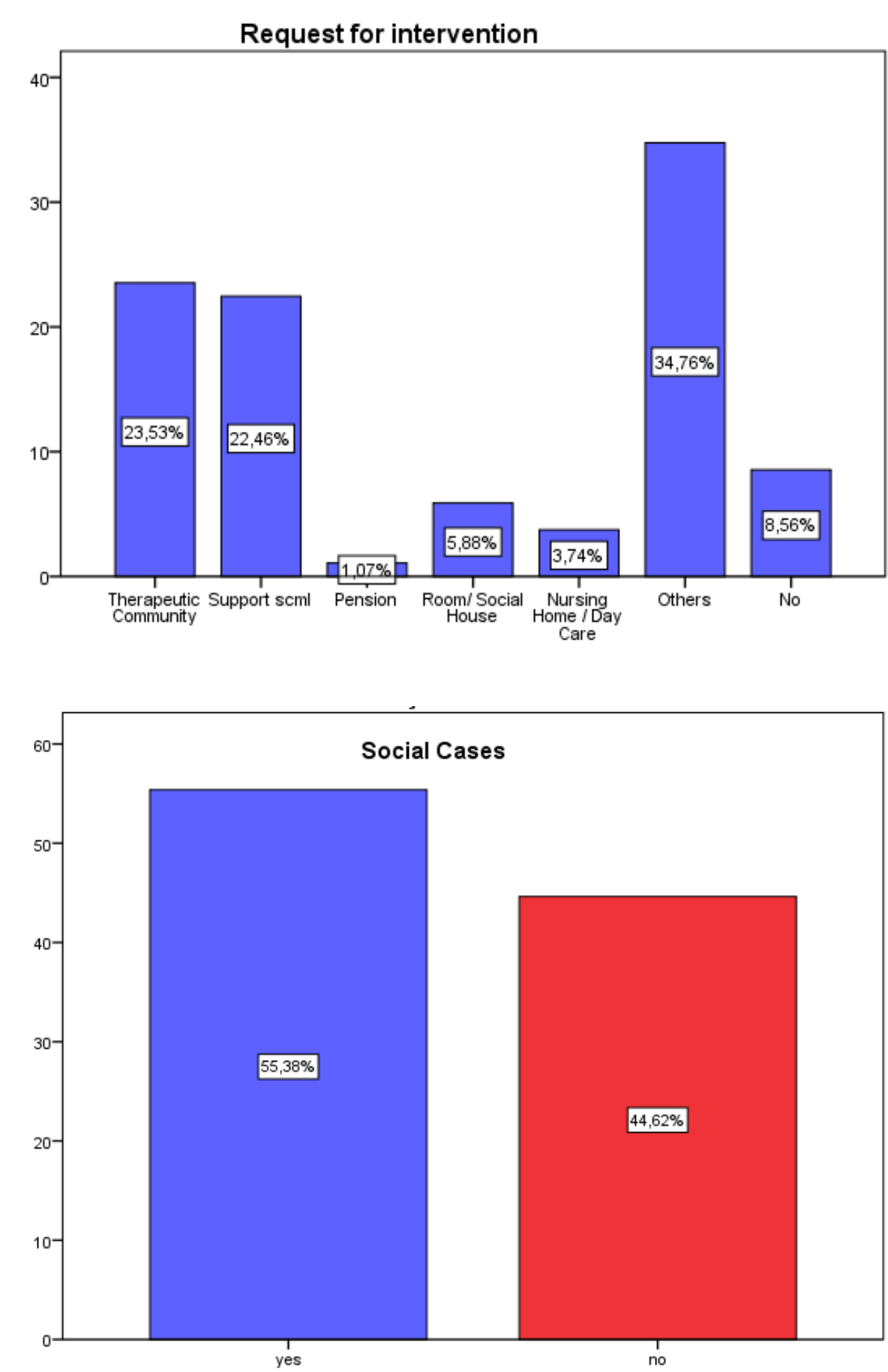

Results: The sample included 188 participants, mostly males $(79,8 \%)$, with a mean age of 49,22 years-old. $54,8 \%$ were considered to have major social problems. More than half of these were male, single, lived alone, and had basic education literacy. They were unemployed (42,0\%) and 46,0\% depended on welfare support.

There was a correlation statistically significant between social situation and the request for intervention of the social worker before living the hospital ( $r=0,061$ e $p=0,024)$.

Of those who ask for support from social services, $23,4 \%$ apply for integration in therapeutic communities, $9,6 \%$ for social or fourth-level housing and $34,6 \%$ it is difficult to fully satisfy their multiple needs, which covers different situations (accommodation, occupation and acquisition of prescribed drugs).

The correlation between the social situation and the economic situation is also statistically significant $(r=-0.289$ and $p=0.00)$, which means that users considered social cases are those who do not have income from work.

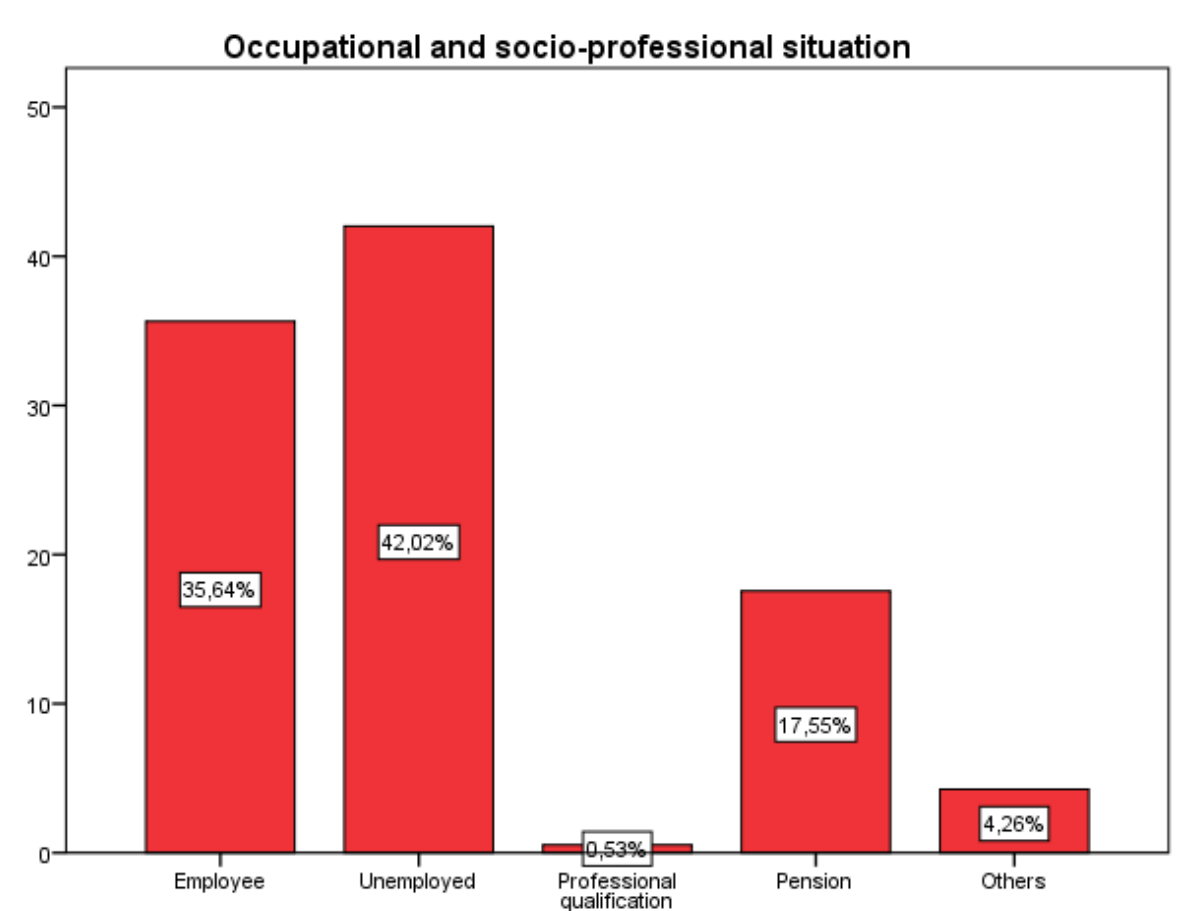

Conclusions: Treatment of patients with alcohol use disorder should include socioeconomic rehabilitation as an important aspect to be taken into account in maintaining abstinence. Treatment units should include multidisciplinary teams so that the diverse needs that patients present can be met. 